\title{
DIFFERENCE EQUATIONS, ISOPERIMETRIC INEQUALITY AND TRANSIENCE OF CERTAIN RANDOM WALKS
}

\author{
$\mathrm{BY}$ \\ JOZEF DODZIUK ${ }^{1}$
}

\begin{abstract}
The difference Laplacian on a square lattice in $\mathbf{R}^{n}$ has been studied by many authors. In this paper an analogous difference operator is studied for an arbitrary graph. It is shown that many properties of the Laplacian in the continuous setting (e.g. the maximum principle, the Harnack inequality, and Cheeger's bound for the lowest eigenvalue) hold for this difference operator. The difference Laplacian governs the random walk on a graph, just as the Laplace operator governs the Brownian motion. As an application of the theory of the difference Laplacian, it is shown that the random walk on a class of graphs is transient.
\end{abstract}

The random walks we consider are defined as follows. Let $K$ be a connected graph (i.e. a one dimensional simplicial complex). For a vertex $x \in K$, let $m(x)$ denote the number of edges emanating from $x$. The probability that a particle moves from $x$ to another vertex $y \in K$ is $1 / m(x)$ if $x$ and $y$ are connected by an edge and it is zero otherwise. As observed by Courant, Friedrichs and Lewy [CFL] for the case of a square lattice in the plane this random walk is intimately related to the difference analog of the Laplacian

$$
\Delta f(x)=\sum_{y \sim x} f(y)-m(x) f(x),
$$

where $y \sim x$ means that $x$ and $y$ are connected by an edge. The operator $\Delta$ defined by $(0.1)$ and its relation to random walks have been studied extensively in the case of a lattice in $\mathbf{R}^{n}$ (cf. e.g. $[\mathbf{C F L}, \mathbf{D u}]$ ). Replacing a lattice by a more general graph corresponds to considering curved manifolds instead of flat ones in the continuous setting. Our results are motivated by [Do], where transience of the Brownian motion on certain manifolds was proved.

In the first section of this paper we show that many familiar facts (the Harnack inequality, the maximum principle, Green's formula, positivity of the first eigenfunction) hold for the discrete Laplacian on an arbitrary graph. In the second section we restrict our attention to graphs which, in a sense, correspond to manifolds of bounded curvature satisfying certain isoperimetric inequality (cf. (2.1) and (2.2)). We show that for such graphs there exists a positive function $f$ defined on vertices for which $\Delta f<0$. Such functions are called superregular in [KSK] (we prefer to call them superharmonic) and their existence implies that the random walk under consideration is transient (cf. [KSK, Chapter 6, §1]), i.e. a particle starting from

Received by the editors August 15, 1983.

1980 Mathematics Subject Classification. Primary 39A10, 60J15; Secondary 58G25.

${ }^{1}$ Research supported in part by NSF Grant No. MCS 8024276. 
a vertex $x$ escapes to infinity with probability one for every vertex $x \in K$. The proofs here are motivated by the proofs of corresponding facts in the smooth case. In order to carry out this analogy we prove a counterpart of Cheeger's inequality [C] for the discrete Laplacian. Finally in $\S 3$ we exhibit many graphs which satisfy the geometric conditions required in $\S 2$.

1. The difference Laplacian. In this section $K$ is an arbitrary graph, i.e. a connected simplicial complex of one dimension. We denote by $C^{0}(K)$ the space of all real valued 0 -cochains, i.e. functions on vertices of $K$. Similarly, $C^{1}(K)$ is the space of all functions $\varphi$ defined on oriented edges of $K$ and satisfying

$$
\varphi([x, y])=-\varphi([y, x])
$$

where $[x, y]$ denotes an oriented (directed) edge beginning at $x$ and ending at $y$. For every edge of $K$ we fix, once and for all, a direction. Nothing will depend on this choice, but it is convenient to make in order to write certain formulae below in an unambiguous way. In what follows an edge will be understood to be an edge with the chosen direction.

Assume now that $K$ is finite. For $f_{1}, f_{2} \in C^{0}(K)$ and $\varphi_{1}, \varphi_{2} \in C^{1}(K)$ we define inner products as follows:

$$
\left(f_{1}, f_{2}\right)=\sum_{x} f_{1}(x) f_{2}(x), \quad\left(\varphi_{1}, \varphi_{2}\right)=\sum_{\sigma} \varphi_{1}(\sigma) \cdot \varphi_{2}(\sigma)
$$

where $x$ ranges over all vertices of $K$, and $\sigma$ runs over the set of all edges of $K$. The coboundary operator

$$
d f([x, y])=f(y)-f(x)
$$

maps $C^{0}(K)$ into $C^{1}(K)$. We define for $f \in C^{0}(K)$,

$$
\Delta f=-d^{*} d f
$$

where $d^{*}$ is the adjoint of $d$ with respect to the inner products (1.1). A simple calculation using (1.1)-(1.3) yields

LEMMA 1.4. For every $f \in C^{0}(K)$

$$
\Delta f(x)=\sum_{y \sim x} f(y)-m(x) f(x),
$$

where $x \sim y$ indicates that $x$ and $y$ are connected by an edge and $m(x)$ is the number of edges emanating from $x$.

REMARK. If $K$ is not finite we define $\Delta$ by the formula above.

DEFINITION 1.5. A cochain $f \in C^{0}(K)$ is called superharmonic at a vertex $x$ if $\Delta f(x) \leq 0$, i.e. if the value $f(x)$ is greater than or equal to the arithmetic mean of the values at neighboring vertices.

The following is an obvious analog of the maximum (minimum) principle.

LEMMA 1.6. Suppose $f \in C^{0}(K)$ is superharmonic at $x \in K$. If for every neighboring vertex $y \sim x f(y) \geq f(x)$, then $f(y)=f(x)$ for $y \sim x$.

The proof is trivial. 
LEMMA 1.7 (HARNACK INEQUALITY). Suppose $f \in C^{0}(K)$ is superharmonic at $x$ and $y$, where $x \sim y$, and $f>0$. Then

$$
\frac{1}{m(y)} f(x) \leq f(y) \leq m(x) f(x) .
$$

Proof. $0 \geq \Delta f(x)=\sum_{z \sim x} f(z)-m(x) f(x)$. Hence $m(x) f(x) \geq \sum_{z \sim x} f(z) \geq$ $f(y)$ because $f \geq 0$. By symmetry $m(y) f(y) \geq f(x)$.

To state the analog of Green's formula we recall the notion of a relative cochain. For a subcomplex $L \subset K, C^{0}(K, L)$ consists of those cochains $f \in C^{0}(K)$ for which $f(x)=0$ whenever $x \in L$. We define the Laplacian $\Delta_{K, L} f$ by the formula $(0.1)$ if $x \notin L$ and $\Delta_{K, L} f(x)=0$ for $x \in L$. Since $C^{0}(K, L) \subset C^{0}(K)$ the inner product and the coboundary are defined for cochains in $C^{0}(K, L)$.

LEMMA 1.8. Let $f, g \in C^{0}(K, L)$. Then

$$
\left(\Delta_{K, L} f, g\right)=-(d f, d g)=\left(f, \Delta_{K, L} g\right) .
$$

ProOF. This can be calculated directly or derived by observing that

$$
\left(\Delta_{K, L} f, g\right)=(\Delta f, g)=-(d f, d f)
$$

since at the vertices $x$, where $\Delta_{K, L} f(x) \neq \Delta f(x), g(x)=0$.

Thus the operator $-\Delta_{K, L}$ on $C^{0}(K, L)$ is selfadjoint and nonnegative. The following lemma is an analog of some familiar facts in the smooth case.

LEMMA 1.9 .

(a) the smallest eigenvalue $\lambda$ of $-\Delta_{K, L}$ is given by $\lambda=\min ((d f, d f) /(f, f))$, where the minimum is taken over $f \in C^{0}(K, L) \backslash\{0\}$. Moreover, if $(d f, d f) /(f, f)=$ $\lambda$, then $\Delta_{K, L} f+\lambda f=0$.

(b) Assume that the pair $(K, L)$ is connected in the sense that every two vertices $x, y \notin L$ can be joined by a chain of edges $\left[x, y_{1}\right],\left[y_{1}, y_{2}\right], \ldots,\left[y_{n-1}, y_{n}\right],\left[y_{n}, y\right]$ so that $y_{i} \notin L$ for $i=1,2,3, \ldots, n$. Then the multiplicity of $\lambda$ is one and we can choose a positive eigencochain of $-\Delta_{K, L}$ belonging to $\lambda$.

Proof. (a) follows trivially from Lemma 1.8. Note that $(f, f)=(|f|,|f|)$. However, for $f \in C^{0}(K, L)$,

$$
\begin{aligned}
(d f, d f) & =\sum_{\sigma=[x, y]}(f(x)-f(y))^{2} \\
& \geq \sum_{\sigma=[x, y]}(|f(x)|-|f(y)|)^{2}=(d|f|, d|f|) .
\end{aligned}
$$

Thus (a) implies that if $f \in C^{0}(K, L)$ belongs to $\lambda$ so does $g=|f|$. Hence $g \geq 0$ and satisfies $\Delta g=-\lambda g \leq 0$. By Lemma 1.6 and by connectedness $g(x)>0$ whenever $x \notin L$. Suppose $f$ takes both positive and negative values in $K$. By connectedness, there exists an edge $[x, y]$ such that $f(x) \cdot f(y)<0$. In this case the inequality in (1.10) is strict, which contradicts Lemma 1.9(a). It follows that $f$ cannot change sign. Now suppose that $\lambda$ is not a simple eigenvalue. Let $f_{1}, f_{2}$ be two linearly independent eigenfunctions of $-\Delta_{K, L}$ belonging to $\lambda$. Choose a vertex $x \notin L$ and consider a linear map $A: \mathbf{R}^{2} \rightarrow \mathbf{R}$ given by $A(a, b)=a f_{1}(x)+b f_{2}(x)$. This mapping has a nontrivial kernel, i.e. there exists a nonzero eigenfunction belonging to $\lambda$ which vanishes at $x$. We saw above that this is impossible. Thus $\lambda$ is a simple eigenvalue. 
Corollary 1.11. Suppose $L_{1} \subset L_{2} \subset K$. Let $\lambda_{i}, i=1,2$, be the smallest eigenvalue of $-\Delta_{K, L_{i}}$. Then $\lambda_{1} \leq \lambda_{2}$.

PROOF. This follows immediately from the characterization of the smallest eigenvalue in Lemma 1.9(a).

2. The analog of Cheeger's inequality and positive superharmonic functions. In this section $M$ denotes an infinite, connected graph. The formalism of $\S 1$ can be applied to every finite subcomplex $K$ of $M$. If $K$ is such a graph, we define $\partial K$, the boundary of $K$, to consist of those vertices $x$ of $K$ for which at least one of the edges meeting at $x$ is not in $K$, and of all edges of $K$ spanned by such vertices.

We make two geometric assumptions on $M$. The first one corresponds to boundedness of the curvature in the Riemannian setting. Namely, we assume that there exists an integer $m>0$ such that

$$
m(x) \leq m
$$

for all vertices $x \in M$.

The second assumption plays the role of an isoperimetric inequality (cf. [Do, $\S 2])$. We require that there exists a constant $\alpha>0$ so that

$$
\alpha V(K) \leq V(\partial K)
$$

for all finite graphs $K \subseteq M$. Here $V(L)$ stands for the number of vertices of $L$.

Theorem 2.3 is an analog of Cheeger's inequality $[\mathbf{C}]$ :

THEOREM 2.3. Suppose (2.1) and (2.2) are satisfied. If $K$ is a finite subcomplex of $M$ such that $(K, \partial K)$ is connected (cf. Lemma 1.9(b)), then the smallest eigenvalue $\lambda$ of $-\Delta_{K, \partial K}$ satisfies $\lambda \geq \alpha^{2} / 2 m$.

Before proving this theorem we will show that it implies that $M$ carries a nonconstant positive superharmonic function (cochain).

THEOREM 2.4. Suppose $M$ satisfies (2.1) and (2.2). There exists a positive function $f \in C^{0}(M)$ and a real number $\lambda>0$ so that $\Delta f+\lambda f=0$. In particular $f$ is positive, superharmonic and nonconstant. Consequently, the random walk on $M$ is transient.

Proof. Fix a vertex $x_{0} \in M$. Define $K_{n}$ to be the complex consisting of those vertices of $M$ which can be joined to $x_{0}$ by a path consisting of at most $n$ edges, together with all edges spanned by these vertices. Clearly $\left(K_{n}, \partial K_{n}\right)$ is connected for $n=1,2, \ldots$, and every finite subcomplex of $M$ is contained in $K_{n}$ for sufficiently large $n$. Let $\lambda_{n}$ be the smallest eigenvalue of $\Delta_{K_{n}, \partial K_{n}}$, and let $f_{n} \in C^{0}\left(K_{n}, \partial K_{n}\right)$ be the corresponding eigencochain normalized so that

$$
f_{n}\left(x_{0}\right)=1 \text {. }
$$

By Corollary 1.11 and Theorem 2.3

$$
\lambda_{n} \geq \lambda_{n+1} \geq \frac{\alpha^{2}}{2 m}
$$

i.e. $\lambda=\lim _{n \rightarrow \infty} \lambda_{n}>0$. The functions $f_{n}$ satisfy $\Delta f_{n}=-\lambda_{n} f_{n}<0$ at interior vertices of $K_{n}$, i.e. are superharmonic. By the Harnack inequality the sequence 
$\left(f_{n}(x)\right)_{n=1}^{\infty}$ is bounded at every vertex $x$ of $M$ (strictly speaking $f_{n}(x)$ is defined only for sufficiently large $n$ ). Using the diagonal process we can find a subsequence $\left(f_{n_{k}}\right)_{k=1}^{\infty}$ such that $\lim _{k \rightarrow \infty} f_{n_{k}}(x)=f(x)$ exists for all $x \in M$. We see that $\Delta f+\lambda f=0$. Moreover, by $(2.5), f\left(x_{0}\right)=1$. Hence $f \geq 0, \Delta f \leq 0$ and $f$ is not constant, since $\Delta f\left(x_{0}\right)=-\lambda f\left(x_{0}\right)=-\lambda<0$. By the maximum principle or by repeated application of the Harnack inequality $f$ is strictly positive everywhere. The transience of the random walk on $M$ is a consequence of Proposition 6.3, Chapter 6 of $[\mathbf{K S K}]$.

PROOF OF THEOREM 2.3 Let $f \in C^{0}(K, \partial K)$ be the eigencochain of $-\Delta_{K, \partial K}$ belonging to the smallest eigenvalue $\lambda$. By Lemma (1.9)(a)

$$
\lambda=(d f, d f) /(f, f) .
$$

Consider the expression $A=\sum_{\sigma=[x, y]}\left|f^{2}(x)-f^{2}(y)\right|$ where the summation is extended over all edges $\sigma$ of $K$. Clearly

$$
\begin{aligned}
A & =\sum|f(x)+f(y)| \cdot|f(x)-f(y)| \\
& \leq\left(\sum|f(x)+f(y)|^{2}\right)^{1 / 2} \cdot\left(\sum|f(x)-f(y)|^{2}\right)^{1 / 2} \\
& \leq \sqrt{2}\left(\sum\left(f^{2}(x)+f^{2}(y)\right)\right)^{1 / 2} \cdot(c f, d f)^{1 / 2} .
\end{aligned}
$$

In $\sum\left(f^{2}(x)+f^{2}(y)\right)$ every vertex contributes as many times as the number of edges emanating from it. Hence, by $(2.1)$,

$$
A \leq \sqrt{2 m}(f, f)^{1 / 2} \cdot(d f, d f)^{1 / 2} .
$$

On the other hand we can estimate $A$ from below in terms of $(f, f)$ as follows. Let $0=\beta_{0}<\beta_{1}<\beta_{2},<\cdots<\beta_{N}$ be the sequence of all values of $f$. Note that, since $\Delta f=-\lambda f<0$, every interior vertex $x$ of $K$ has a neighbor $y$ such that $f(y)<f(x)$. Define $L_{i}, i=0,1, \ldots, N$, as follows. A vertex $x$ of $K$ is in $L_{i}$ if $f(x) \geq \beta_{i}$. An edge belongs to $L_{i}$ if both endpoints are in $L_{i}$. Now

$$
A=\sum_{i=1}^{N} \sum_{f(x)=\beta_{i}} \sum_{y \sim x, f(y)<\beta_{i}}\left(f^{2}(x)-f^{2}(y)\right) .
$$

If $f(x)=\beta_{i}$ and $f(y)=\beta_{i-k}$ for some $k \in\{1,2, \ldots, i\}$, then, on the one hand, $f^{2}(x)-f^{2}(y)=\beta_{i}^{2}-\beta_{i-k}^{2}=\left(\beta_{i}^{2}-\beta_{i-1}^{2}\right)+\left(\beta_{i-1}^{2}-\beta_{i-2}^{2}\right)+\cdots+\left(\beta_{i-k+1}^{2}-\beta_{i-k}^{2}\right)$ and, on the other hand, $x \in \partial L_{i} \cap \partial L_{i-1} \cap \cdots \cap \partial L_{i+1-k}$. It follows that

$$
A=\sum_{i=1}^{N} \sum_{x \in \partial L_{i}}\left(\beta_{i}^{2}-\beta_{i-1}^{2}\right)=\sum_{i=1}^{N} V\left(\partial L_{i}\right)\left(\beta_{i}^{2}-\beta_{i-1}^{2}\right) .
$$

Applying (2.2) we obtain

$$
A \geq \alpha \sum_{i=1}^{N} V\left(L_{i}\right)\left(\beta_{i}^{2}-\beta_{i-1}^{2}\right) .
$$

"Summation by parts" yields now

$$
A \geq \alpha\left\{V\left(L_{N}\right) \beta_{N}^{2}+\sum_{i=1}^{N-1} \beta_{i}^{2}\left(V\left(L_{i}\right)-V\left(L_{i+1}\right)\right)\right\} .
$$


A vertex $x \in L_{i} \backslash L_{i+1}$ if and only if $f(x)=\beta_{i}$. Therefore $A \geq \alpha(f, f)$. This inequality combined with $(2.7)$ yields

$$
\lambda=\frac{(d f, d f)}{(f, f)} \geq \frac{\alpha^{2}}{2 m}
$$

which proves the theorem.

REMARK. The proof above is patterned after the proof of Cheeger's inequality in $[\mathbf{C}]$. The expression $A$ plays the role of $\left|d\left(f^{2}\right)\right|$ and the estimate (2.7) is analogous to

$$
\left|d\left(f^{2}\right)\right| \leq 2 f|d f| \leq 2\left(|f|^{2}\right)^{1 / 2} \cdot\left(|d f|^{2}\right)^{1 / 2} .
$$

Finally, the inequality $\alpha V(L) \leq V(\partial L)$ enters into the proof in the way the analogous isomperimetric inequality enters into Cheeger's proof.

3. An isoperimetric inequality. Suppose $S$ is a simply connected Riemannian surface of curvature bounded from above by a negative constant. It is well known that for every open, relatively compact subset $D$ of $S$ with smooth boundary

$$
\alpha A(D) \leq L(\partial D),
$$

where $\alpha$ is a constant independent of $D, A$ stands for area and $L$ denotes length. We shall derive a combinatorial analog of (3.1) and use it to exhibit many graphs satisfying hypotheses of Theorem 2.4 .

Let $N$ be an oriented, triangulated, open surface without boundary. For a finite subcomplex $L$ of $N$, let $V(L), E(L), F(L)$ denote the number of vertices, edges and faces, respectively, of $L$. For such a subcomplex $L$ define $\delta L$ to be the graph consisting of those edges and vertices of $L$ which are on the boundary of a triangle not in $L$.

PROPOSITION 3.2. Suppose $N$ is as above and suppose that $m(x) \geq 7$ for every vertex $x \in N$. If $N$ is planar $F(L) \leq 26 E(\delta L)$ for every finite subcomplex $L$ of $N$.

REMARK. The condition $m(x) \geq 7$ is the combinatorial analog of negative curvature bounded away from zero. Planarity means that every cycle on $N$ disconnects. Equivalently (cf. [AhS, Chapter III, §4]) $N$ is homeomorphic to a subset of the plane.

ProOF OF Proposition 3.2. We want to prove the inequality $F(L) \leq$ $26 E(\delta L)$. If $L$ has edges or vertices which do not lie on the boundary of a triangle in $L$, we can remove those edges and vertices and prove the inequality for the resulting complex. Therefore we assume, with no loss of generality, that if a vertex on an edge belongs to $L$, then one of the adjacent triangles does too. Since $N$ is planar, we can assume that $L$ is contained in the sphere $S^{2}$. The Euler characteristic $\chi(L)$ satisfies

$$
\chi(L)=V(L)-E(L)+F(L)=2-n,
$$

where $n$ is the number of components of $S^{2} \backslash L$. Clearly $n \leq E(\delta L)$ and

$$
E(\delta L) \geq n-2=E(L)-F(L)-V(L) .
$$

On the other hand, since $m(x) \geq 7$ for all vertices $x \in N$,

$$
3 F(L) \geq 7 V_{i}(L)+V(\delta L),
$$


where $V_{i}(L)$ denotes the number of interior vertices of $L$. Since $V(L)=V_{i}(L)+$ $V(\delta L)$, we obtain

$$
F(L) \geq \frac{7}{3} V(L)-2 V(\delta L)
$$

Clearly $\delta L$ consists of circles (polygons to be exact) possibly touching at some vertices. It follows that $V(\delta L) \leq E(\delta L)$. Substituting this into (3.4) yields

$$
V(L) \leq \frac{3}{7} F(L)+\frac{6}{7} E(\delta L)
$$

Moreover, if $E_{i}(L)$ denotes the number of interior edges of $L$,

$$
3 F(L)=2 E_{i}(L)+E(\delta L) \leq 2 E(L) .
$$

Together with (3.3) and (3.5) this gives

$$
E(\delta L) \geq-F(L)-\frac{3}{7} F(L)-\frac{6}{7} E(\delta L)+\frac{3}{2} F(L),
$$

i.e. $26 E(\delta L) \geq F(L)$.

REMARK. The method of proof of the proposition above is borrowed from [DB] where an analogous inequality is proved in a somewhat different setting.

COROLLARY 3.6. Let $N$ be a triangulated planar surface with the property that $m \geq m(x) \geq 7$ at every vertex $x$ of $N$, for an integer $m$ independent of $x$. Then $V(\partial K) \geq V(K) / 78 m$ for every finite graph $K$ contained in $N$.

Proof. Given $K$ define $L$ to be the smallest complex containing $K$ and all the triangles of $N$ all of whose edges are in $K$. According to the definitions of $\partial K$ (cf. beginning of $\S 2$ ) and $\delta L$, the set of vertices of $\partial K$ coincides with the set of vertices of $\delta L$. We can break $L$ up into three parts, $L=L_{0} \cup L_{1} \cup L_{2}$, as follows. $L_{0}$ is the set of isolated vertices of $L$ (i.e. of vertices $x$ such that all the edges meeting at $x$ are not in $L$, hence not in $K) . L_{1}$ consists of all edges of $K$ which are not on the boundary of any triangle of $L$, and $L_{2}$ is the remaining part of $L$.

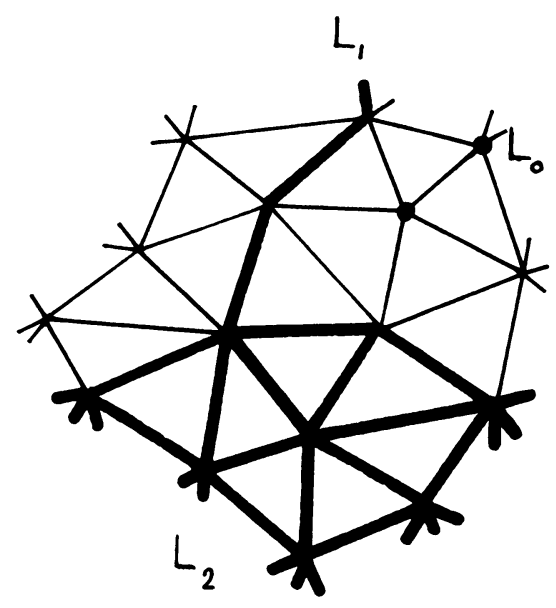

FIGURE 1 
Clearly $V\left(L_{2}\right) \leq 3 F\left(L_{2}\right)$. By Proposition $3.2 F\left(L_{2}\right) \leq 26 E\left(\delta L_{2}\right)$ so that

$$
V\left(L_{2}\right) \leq 78 E\left(\delta L_{2}\right) .
$$

Moreover $2 E\left(\delta L_{2}\right) \leq m V\left(\delta L_{2}\right)$ and, therefore

$$
V\left(L_{2}\right) \leq 39 m V\left(\delta L_{2}\right) .
$$

Obviously $\partial K=L_{0} \cup L_{1} \cup \delta L_{2}$ and $L_{1}$ can have some vertices in common with $\delta L_{2}$. Therefore

$$
V(\partial K) \geq \frac{1}{2}\left(V\left(L_{0}\right)+V\left(L_{1}\right)+V\left(\delta L_{2}\right)\right) .
$$

Similarly, since the sets of vertices of $K$ and $L$ are equal,

$$
V(K)=V(L) \leq V\left(L_{0}\right)+V\left(L_{1}\right)+V\left(L_{2}\right) .
$$

(3.8), (3.9) and (3.10) yield $V(\partial K) \geq V(K) / 78 m$, which completes the proof.

REMARK. Triangulations satisfying assumptions of Corollary 3.6 (and, hence, satisfying (2.1) and (2.2)) occur naturally on planar surfaces which cover compact, oriented surfaces without boundary of genus $g>1$. The simplest example is the plane (realized as the hyperbolic plane) with a tesselation into regular (in the sense of hyperbolic geometry) octagons. To manufacture a triangulation we subdivide every octagon as shown in Figure 2. The resulting triangulation has two classes of vertices with $m(x)$ equal to 8 and 16 respectively since eight octagons meet at every vertex of the original tesselation.

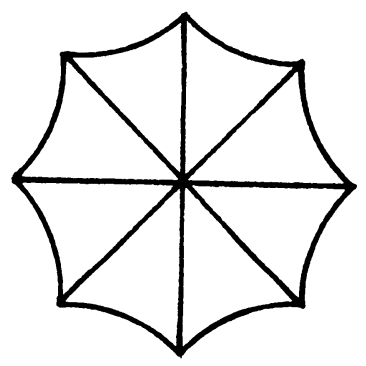

FIGURE 2

\section{REFERENCES}

[AhS] L. V. Ahlfors and L. Sario, Riemann surfaces, Princeton Univ. Press, Princeton, N.J., 1974.

[C] J. Cheeger, A lower bound for the lowest eigenvalue of the Laplacian, Problems in Analysis, A Symposium in honor of S. Bochner, Princeton Univ. Press, Princeton, N.J., 1970, pp. 195199.

[CFL] R. Courant, K. Friedrichs and H. Lewy, Über die partiellen Differenzengleichungen der mathematischen Physik, Math. Ann. 100 (1928), 32-74.

[DB] D. DeBaun, $L^{2}$-cohomology of noncompact surfaces, Trans. Amer. Math. Soc. (to appear).

[Do] J. Dodziuk, Every covering of a compact Riemann surface of genus greater than one carries a nontrivial $L^{2}$ harmonic differential, Acta Math. (to appear).

[Du] R. Duffin, Discrete potential theory, Duke Math. J. 20 (1953), 233-251.

[KSK] J. G. Kemeny, J. L. Snell and A. W. Knapp, Denumerable Markov chains, 2nd ed., SpringerVerlag, New York, 1976.

Department of Mathematics, Queens College, CUny, Flushing, New York 